\title{
AUTOLOGOUS TRANSPLANT IN LYMPHOMAS IN THE CURRENT ERA OF IMMUNOTHERAPY
}

Autologous stem cell transplant (ASCT) is the treatment of choice for aggressive, relapsed/refractory non-Hodgkin's and Hodgkin's lymphomas (HLs). ${ }^{[1,2]}$ In patients aged $<65$ years, $40-45 \%$ achieve cure with this form of treatment. The duration of remission post-transplant depends on the histological subtype, prior treatment and the response to salvage therapy. In Pakistan, the common lymphomas for which patients receive treatment include diffuse large B-cell lymphoma (DLBCL), mantle cell lymphoma, follicular lymphoma and HL. ${ }^{[3]}$ Of these, ASCT is commonly performed for DLBCL and HL in the second complete remission. ${ }^{[4]}$

Based on the opinion that ASCT can potentially cure $50 \%$ of patients who relapse but is chemosensitive, this procedure was performed in a cohort of patients who partially responded to chemotherapy. Philip et al., in $1998,{ }^{[5]}$ reported that $76 \%$ of the patients remained disease free with a 6 -year survival of $75 \%$ post-ASCT. The Non-HL Cooperative Study in 1993 showed a 5 -year disease-free survival of $36 \%(n=21$ patients $){ }^{[6]}$ This translated as a potential cure of approximately one in three patients with partial remission after ASCT. Hamadani et al., in 2014, ${ }^{[7]}$ reported important outcomes on $n=516$ DLBCL patients with respect to Rituximabbased therapies and ASCT. The observations included as follows: (a) Patients receiving Rituximab-based therapy and experiencing late relapse ( $>1$ year after treatment) have excellent response to ASCT; (b) in patients with early relapse (within 1 year of treatment with Rituximabbased regimens), ASCT can confer durable disease control and (c) in patients who have refractory disease but respond to salvage therapies, ASCT is the only potentially curative treatment option; however, a fourth observation included that a subset of patients relapsed within 6-9 months of transplant. This led to the inference of maintenance Rituximab. Gisselbrecht et al., in 2012, ${ }^{[8]}$ after performing ASCT and randomly assigning patients to two groups of cohort $(n=122$ patients who received

Correspondence: Natasha Ali, Department of Pathology and Laboratory Medicine/Oncology, Aga Khan University, Karachi, Pakistan. E-mail: natasha.ali@aku.edu
Rituximab and $n=120$ patients who did not receive Rituximab) concluded that there was no difference between the maintenance group and control group and did not recommend maintenance post-transplant. A study was done by Zhang et al. ${ }^{\left[{ }^{[0]}\right.}$ demonstrated that ASCT in combination with Rituximab in vivo purging followed by maintenance Rituximab prolongs progression-free survival and overall survival. However, this was a cohort of $n=12$ young patients with DLBCL. Therefore, the current recommendation for relapsed/refractory DLBCL remains to be ASCT in transplant-eligible patients with no role of maintenance Rituximab.

Most patients with HL achieve long-term remission with chemo or radiotherapy. However, $10-30 \%$ of patients progress or relapse depending on the stage. ASCT can cure $50 \%$ of these patients. In the remaining $50 \%$, treatment of $\mathrm{HL}$ remains to be a challenge. ${ }^{[10,11]}$ The use of brentuximab vedotin (anti-CD30) in early Phase I trials did not demonstrate any clinical significance. ${ }^{[12,13]}$ Phase II trials, however, demonstrated satisfactory results. The German Hodgkin Study Group showed an overall response rate (ORR) of $60 \%$ in $n=45$, heavily pre-treated patients. ${ }^{[14]}$ Due to this increased ORR and tolerable safety profile of the drug, Food and Drug Administration granted an accelerated approval for the treatment of HL in patients who relapse after ASCT or in patients ineligible for transplant. The National Comprehensive Cancer Network guideline includes brentuximab for the same. Brentuximab has also been used as a bridge to ASCT in patients who fail standard and salvage treatments. ${ }^{[15]}$

Moving forward, to further improve outcomes after ASCT in relapsed/refractory lymphomas, efforts need to be focused on evaluating novel consolidation or maintenance strategies, possibly with agents not used in induction/ salvage therapies. The current standard of care remains to be high-dose chemotherapy followed by ASCT and immunotherapies cannot be used as an alternative to transplant. 


\section{Natasha Ali $^{1}$, Mohammad Khurshid ${ }^{2}$ \\ ${ }^{1}$ Department of Pathology and Laboratory Medicine/Oncology, Aga Khan University, Karachi, Pakistan, ${ }^{2}$ Department of Oncology, Aga Khan \\ University, Karachi, Pakistan}

Received: 19 October 2016 / Accepted: 26 November 2016

\section{References}

1. Philip T, Guglielmi C, Hagenbeek A, et al. Autologous bone marrow transplantation as compared with salvage chemotherapy in relapses of chemotherapy-sensitive nonhodgkin's lymphoma. N Engl J Med 1995;333:1540-5.

2. Philip T, Armitage JO, Spitzer G, et al. High-dose therapy and autologous bone marrow transplantation after failure of conventional chemotherapy in adults with intermediategrade or high-grade non-Hodgkin's lymphoma. N Engl J Med 1987;316:1493-8.

3. Pervez S. Non-hodgkin lymphoma (NHL) in Pakistan. Int J Mol Cell Med 2012;1:62-3.

4. Ali N, Adil SN, Shaikh MU. Autologous hematopoietic stem cell transplantation-10 years of data from a developing country. Stem Cells Transl Med 2015;4:873-7.

5. Philip T, Hartmann O, Biron P, et al. High-dose therapy and autologous bone marrow transplantation in partial remission after first-line induction therapy for diffuse non-Hodgkin's lymphoma. J Clin Oncol 1988;6:1118-24.

6. Santini G, Congiu AM, Coser P, et al. Autologous bone marrow transplantation in 100 cases of poorprognosis non-Hodgkin's lymphoma. A report of the Non-Hodgkin's lymphoma cooperative study group. In: Dicke KA, Keating A, editors. Autologous Bone Marrow Transplantation. Arlington (Texas): Proceeding of the Sixth International Symposium; 1993. p. 75-82.

7. Hamadani M, Hari PN, Zhang Y, et al. Early failure of frontline rituximab-containing chemo-immunotherapy in diffuse large B cell lymphoma does not predict futility of autologous hematopoietic cell transplantation. Biol Blood Marrow Transplant 2014;20:1729-36.

8. Gisselbrecht C, Schmitz N, Mounier N, et al. Rituximab maintenance therapy after autologous stem-cell transplantation in patients with relapsed CD20(+) diffuse large B-cell lymphoma: Final analysis of the collaborative trial in relapsed aggressive lymphoma. J Clin Oncol 2012;30:4462-9.

9. Zhang W, Jiao L, Zhou DB, et al. Rituximab purging and maintenance therapy combined with autologous stem cell transplantation in patients with diffuse large B-cell lymphoma. Oncol Lett 2010;1:733-8.

10. Majhail NS, Weisdorf DJ, Defor TE, et al. Long-term results of autologous stem cell transplantation for primary refractory or relapsed Hodgkin's lymphoma. Biol Blood Marrow Transplant 2006;12:1065-72.

11. Josting A, Müller H, Borchmann P, et al. Dose intensity of chemotherapy in patients with relapsed Hodgkin's lymphoma. J Clin Oncol 2010;28:5074-80.

12. Bartlett NL, Younes A, Carabasi MH, et al. A phase 1 multidose study of SGN-30 immunotherapy in patients with refractory or recurrent $\mathrm{CD} 30+$ hematologic malignancies. Blood 2008;111:1848-54.

13. Forero-Torres A, Leonard JP, Younes A, et al. A phase II study of SGN-30 (anti-CD30 mAb) in Hodgkin lymphoma or systemic anaplastic large cell lymphoma. Br J Haematol 2009;146:171-9.

14. Rothe A, Sasse S, Goergen H, et al. Brentuximab vedotin for relapsed or refractory $\mathrm{CD} 30$ + hematologic malignancies: The German Hodgkin study group experience. Blood 2012;120:1470-2.

15. Sasse $\mathrm{S}$, Rothe A, Goergen $\mathrm{H}$, et al. Brentuximab vedotin (SGN-35) in patients with transplant-naive relapsed/refractory Hodgkin lymphoma. Leuk Lymphoma 2013;54:2144-8. 